\title{
Physalin B not only inhibits the ubiquitin-proteasome pathway but also induces incomplete autophagic response in human colon cancer cells in vitro
}

\author{
Yi-ming MA, Wei HAN, Jia LI, Li-hong HU*, Yu-bo ZHOU* \\ National Center for Drug Screening, Shanghai Institute of Materia Medica, Shanghai Institutes for Biological Sciences, Chinese \\ Academy of Sciences, Shanghai 201203 , China
}

\begin{abstract}
Aim: To investigate the effects of physalin B insolated from Physalis divericata on human colon cancer cells in vitro and its anticancer mechanisms.

Methods: Human HCT116 colon cancer cell line was tested. Cell viability and apoptosis were detected, and relevant proteins were measured using Western blot analyses. Autophagosomes were observed in stable GFP-LC3 HCT116 cells. Localization of autophagosomes and lysosomes was evaluated in GFP-LC3/RFP-LAMP1-co-transfected cells. Microtubules and F-actin microfilaments were observed with confocal microscope. Mitochondrial ROS (mito-ROS) was detected with flow cytometry in the cells stained with MitoSox dye.

Results: Physalin B inhibited the viability of HCT116 cells with an $\mathrm{IC}_{50}$ value of $1.35 \mu \mathrm{mol} / \mathrm{L}$. Treatment of the cells with physalin B (2.5-10 $\mu \mathrm{mol} / \mathrm{L})$ induced apoptosis and the cleavage of PARP and caspase-3. Meanwhile, physalin B treatment induced autophagosome formation, and accumulation of LC3-II and p62, but decreased Beclin 1 protein level. Marked changes of microtubules and F-actin microfilaments were observed in physalin B-treated cells, which led to the blockage of co-localization of autophagosomes and lysosomes. Physalin B treatment dose-dependently increased the phosphorylation of p38, ERK and JNK in the cells, whereas the p38 inhibitor SB202190, ERK inhibitor U0126 or JNK inhibitor SP600125 could partially reduce physalin B-induced PARP cleavage and p62 accumulation. Moreover, physalin B treatment dose-dependently increased mito-ROS production in the cells, whereas the ROS scavenger NAC could reverse physalin B-induced effects, including incomplete autophagic response, accumulation of ubiquitinated proteins, changes of microtubules and F-actin, activation of p38, ERK and JNK, as well as cell death and apoptosis.

Conclusion: Physalin B induces mito-ROS, which not only inhibits the ubiquitin-proteasome pathway but also induces incomplete autophagic response in HCT116 cells in vitro.
\end{abstract}

Keywords: physalin B; Physalis angulata L; HCT116 colon cancer cells; autophagy; reactive oxygen species; p38 mitogen-activated protein kinases; microtubule-associated proteins; apoptosis; ubiquitins; $N$-acetyl-L-cysteine

Acta Pharmacologica Sinica (2015) 36: 517-527; doi: 10.1038/aps.2014.157; published online 23 Mar 2015

\section{Introduction}

Nature is a still largely unexplored source of new therapeutic candidate compounds due to the huge chemical diversity found in plants, animals, marine organisms and microorganisms ${ }^{[1]}$. Natural bioactive molecules have also been presumed to be safer than synthesized chemical compounds ${ }^{[2]}$. Many of these compounds have been identified as potential anticancer agents $^{[3-10]}$. Physalis angulata L (Solanaceae) is a plant widely distributed throughout the tropical and subtropical regions of

\footnotetext{
* To whom correspondence should be addressed.

E-mail ybzhou@simm.ac.cn (Yu-bo ZHOU); Inhu@simm.ac.cn (Li-hong HU)

Received 2014-09-11 Accepted 2014-12-08
}

the world. Extracts or infusions of this plant have been used in various countries in popular medicine as treatment for a variety of illnesses, such as malaria, asthma, hepatitis, dermatitis and rheumatism ${ }^{[11]}$. Several studies have investigated the anticancer potential of $P$ angulata, which showed that the organic extracts and some purified compounds possess cytotoxic activity against several tumor cell lines and sarcoma 180 tumor cells transplanted into mice ${ }^{[12]}$. The cytotoxicity of this plant has been attributed to the physalins, an inhibitor of the ubiquitin-proteasome pathway (UPP) ${ }^{[13]}$.

The UPP and autophagy-lysosome pathway (ALP) are the two major systems for eukaryotic intracellular protein clearance that are functionally coupled to maintain homeostasis ${ }^{[14,15]}$. The UPP is responsible for the degradation 
of short-lived proteins, whereas the ALP is responsible for the degradation of most long-lived proteins and some organelles. Proteasome inhibitors, such as Bortezomib, have high clinical activity in hematological tumors ${ }^{[16,17]}$, and autophagy inhibitors, including 3-MA, are also being evaluated as potential antitumor agents ${ }^{[18]}$. In addition, the combined inhibition of UPP and ALP would cause more cancer cell death than blocking either degradation pathway alone ${ }^{[19,20]}$.

In the present study, we show that in contrast with other reported UPP inhibitors, physalin B induces an incomplete autophagic response along with blocked lysosome degradation. Further analysis shows that the inhibition of the two degradation pathways and apoptosis are dependent on mitoROS generation.

\section{Materials and methods Reagents}

Hyclone FBS (Cat \#SH30071.01) and ECL Western blotting detection reagents (Cat \#RPN2108) were purchased from GE Healthcare Life Sciences (Logan, UT, USA). Penicillin/ Streptomycin (Cat \#15140-122) was purchased from Invitrogen Corporation (Carlsbad, CA, USA). McCoy's 5A Modified Medium (Cat \#M4892), G418 (Cat \#A1720), rapamycin (Cat \#R8781), $\mathrm{H}_{2} \mathrm{O}_{2}$ (Cat \#323381), MTT Formazan (Cat \#M2003), paraformaldehyde (Cat \#P6148), Sulforhodamine B (Cat \#S1402), SB 202190 (Cat \#S7076), U0126 (Cat \#U120), SP600125 (Cat \#S5567) and N-acetyl-L-cysteine (Cat \#A7250) were purchased from Sigma-Aldrich (St Louis, MO, USA). Fluorescein phalloidin (Cat \#F432) and MitoSOX ${ }^{\mathrm{TM}}$ Red mitochondrial superoxide indicator (Cat \#M36008) were purchased from Life Technologies (Grand Island, NY, USA). Complete protease inhibitor cocktail (Cat \#11697498001) was ordered from Roche Diagnostics Corporation (Indianapolis, IN, USA).

Physalin B was isolated from the EtOH-extract of the aerial part of Physalis divericata, as previously described ${ }^{[21]}$. The structure of physalin B was identified by comparison of its mass spectrometry and nuclear magnetic resonance data with those described in the literature ${ }^{[22]}$. The purity of physalin B was determined to be $>98 \%$ by high performance liquid chromatography. For the present studies, physalin B was solubilized in DMSO to achieve a stock solution concentration of 10 $\mathrm{mmol} / \mathrm{L}$.

Anti-PARP (Cat \#9542), anti-caspase-3 (Cat \#9662), antiBeclin 1 (Cat \#4122), anti-phospho-mTOR (Ser2448) (Cat \#2971), anti-LC3A/B (Cat \#4108), anti-phospho-p38 MAPK (Thr180/Tyr182) (Cat \#4511), anti-p38 MAPK (Cat \#9212), anti-phospho-SAPK/JNK (Thr183/Tyr185) (Cat \#9255), antiSAPK/JNK (Cat \#9258), anti-phospho-p44/42 MAPK (Erk1/2) (Cat \#9101), anti-p44/42 MAPK (Erk1/2) (Cat \#4695), antiUbiquitin (Cat \#3936), and anti- $\beta$-actin (Cat \#8457) antibodies were purchased from Cell Signaling Technology (Boston, MA, USA). Anti-p62/SQSTM1 antibody (Cat \#ab56416) was purchased from Abcam Inc (Cambridge, MA, USA). Anti- $\beta$ tubulin (Cat \#T-4026) was purchased from Sigma Aldrich (St Louis, MO, USA). Peroxidase-affiniPure goat anti-rabbit IgG (Code: 112-035-175) and goat anti-mouse IgG (Code: 115-035-
174) were purchased from Jackson Immuno Research Laboratories Inc (Baltimore, MD, USA). Hoechst 33342 dye (Cat \#H3570) and Alexa Fluor 488 dye-labeled donkey anti-mouse IgG (Cat \#A-21202) were purchased from Invitrogen Corporation (Carlsbad, CA, USA).

\section{Cell lines and cell culture}

The human HCT116 colon cancer cell line was ordered from the Cell Bank (Chinese Academy of Sciences, Shanghai, China). HCT116 and engineered HCT116 GFP-LC3 cells were cultured in McCoy's 5A modified medium supplemented with $10 \% \mathrm{FBS}$ and $50 \mu \mathrm{g} / \mathrm{mL}$ penicillin/streptomycin. The cells were kept in a humidified atmosphere of $5 \% \mathrm{CO}_{2}$ and $95 \%$ air at $37^{\circ} \mathrm{C}$.

\section{Generation of stably transfected HCT116 GFP-LC3 cell lines}

HCT116 cells were transfected with the plasmid encoding GFP-LC3, and the stable cell lines were selected in McCoy's 5A Modified Medium with 10\% FBS containing $500 \mu \mathrm{g} / \mathrm{mL}$ G418. The stable cell line expressing GFP-LC3 was treated with 10 $\mu \mathrm{mol} / \mathrm{L}$ physalin B for $12 \mathrm{~h}$. The translocation of GFP-LC3 from the cytosol to autophagic vacuoles was observed under an Olympus Fluoview FV1000 confocal microscope (Olympus, Tokyo, Japan).

\section{MTT viability assay}

The inhibitory effect of physalin B on the growth of HCT116 cells was evaluated using the MTT viability assay as described previously ${ }^{[23]}$.

Briefly, the cells were seeded onto plastic 96-well cell culture plates and cultured at $37^{\circ} \mathrm{C}$. After $24 \mathrm{~h}$, physalin B with doses ranging from $10 \mu \mathrm{mol} / \mathrm{L}$ to $0.31 \mu \mathrm{mol} / \mathrm{L}$ at a dilution ratio of 1:2 were added, and the cells were further incubated for $72 \mathrm{~h}$. MTT was then added to each well at a final concentration of 1 $\mathrm{g} / \mathrm{L}$. After a $3 \mathrm{~h}$ incubation at $37^{\circ} \mathrm{C}$, the medium was gently discarded and DMSO was added. The optical density was determined at $550 \mathrm{~nm} / 690 \mathrm{~nm}$ using the VersaMax Microplate Reader (Molecular Devices). The experiments were performed in triplicate. The $\mathrm{IC}_{50}$ values were derived from a nonlinear regression model (curvefit) based on a sigmoidal dose response curve (variable slope) and computed using GraphPad Prism (Version 5.02, GraphPad Software Inc, San Diego, CA, USA). The data were expressed as the mean \pm SEM.

\section{Western blotting analysis}

The cells were treated with varying doses of physalin B for different time points. The cells were lysed with cell lysis buffer $(1 \% \mathrm{NP}-40,150 \mathrm{mmol} / \mathrm{L} \mathrm{NaCl}, 20 \mathrm{mmol} / \mathrm{L}$ Tris- $\mathrm{HCl}, 1$ $\mathrm{mmol} / \mathrm{L}$ EDTA, $1 \mathrm{mmol} / \mathrm{L}$ EGTA and complete protease inhibitor cocktail). The lysates were fractionated by SDSPAGE and transferred onto Hybond-C nitrocellulose membranes (Cat \#RPN203E, GE Life Sciences, Pittsburgh, PA, USA) and immunoblotted as described previously ${ }^{[24]}$. The immunoreactive bands were detected using ECL Western blotting detection reagents (Cat\#RPN2108, GE Life Sciences, Pittsburgh, PA, USA). 


\section{Co-localization analysis}

The effect of physalin B on the co-localization of autophagosomes and lysosomes was evaluated in GFP-LC3/RFP-LAMP1 co-transfected HCT116 cells. Briefly, the cells were transfected with GFP-LC3 and RFP-LAMP1 plasmids using lipofectamine 2000 reagent (Cat \#11668019, Invitrogen, Grand Island, NY, USA). The cells were treated with $0.1 \%$ DMSO (negative control), $5 \mu \mathrm{mol} / \mathrm{L}$ rapamycin (an autophagy inducer) and 10 $\mu \mathrm{mol} / \mathrm{L}$ physalin B for $12 \mathrm{~h}$, followed by observation using the Olympus Fluoview FV1000 confocal microscope (Olympus, Tokyo, Japan).

\section{Microtubule observation}

The microtubules were observed using an immunocytochemistry assay ${ }^{[25]}$. Briefly, the cells were grown on glass coverslips for $24 \mathrm{~h}$, treated with $10 \mu \mathrm{mol} / \mathrm{L}$ physalin B for $12 \mathrm{~h}$, fixed using $4 \%$ paraformaldehyde for $20 \mathrm{~min}$, incubated with $0.1 \%$ Triton X-100 for $10 \mathrm{~min}$, blocked with 5\% BSA for $1 \mathrm{~h}$ at room temperature, and incubated with a monoclonal $\beta$-tubulin antibody overnight at $4{ }^{\circ} \mathrm{C}$. The cells were then washed three times with $1 \times$ PBS and incubated with Alexa Fluor ${ }^{\circledR} 488$ labeled donkey anti-Mouse IgG at room temperature for $1 \mathrm{~h}$. The coverslips were washed and photographed using the Olympus Fluoview FV1000 confocal microscope (Olympus, Tokyo, Japan).

\section{F-actin microfilament observation}

The F-actin microfilaments were observed by staining with fluorescein phalloidin according to the manufacturer's instructions. Briefly, the cells were grown on glass coverslips for $24 \mathrm{~h}$, treated with $10 \mu \mathrm{mol} / \mathrm{L}$ physalin B for $12 \mathrm{~h}$, fixed using $4 \%$ paraformaldehyde for $10 \mathrm{~min}$, incubated with $0.1 \%$ Triton X-100 for $5 \mathrm{~min}$, and then stained with fluorescein phalloidin for $20 \mathrm{~min}$. The coverslips were washed and photographed using the Olympus Fluoview FV1000 confocal microscope (Olympus, Tokyo, Japan).

\section{Measurements of mito-ROS level}

The level of mito-ROS was measured using the MitoSox ${ }^{\mathrm{TM}}$ Red mitochondrial superoxide indicator (Cat \#M36008, Life Technologies, Grand Island, NY, USA) according to the manufacturer's instructions. Briefly, the cells were treated with physalin B for $4 \mathrm{~h}$, collected upon trypsin treatment, washed twice with ice cold $1 \times$ PBS, and incubated with $5 \mu \mathrm{mol} / \mathrm{L}$ MitoSOX for $20 \mathrm{~min}$ in the dark. The level of mito-ROS was measured using a BD FACSCalibur Flow Cytometer and analyzed using FCS Express V3 software (BD Biosciences, San Jose, CA, USA).

\section{Sulforhodamine B (SRB) viability assay}

The SRB assay was used to evaluate the effect of the ROS scavenger $N$-acetyl-L-cysteine (NAC) on the growth of physalin B-treated cells. Briefly, the cells (3000 cells per well) were seeded into 96 -well plates and cultured at $37^{\circ} \mathrm{C}$. After $24 \mathrm{~h}$, physalin $\mathrm{B}$ at final concentrations ranging from $10 \mu \mathrm{mol} / \mathrm{L}$ to $0.31 \mu \mathrm{mol} / \mathrm{L}$ at a dilution ratio of 1:2 with or without NAC (final concentration: $10 \mathrm{mmol} / \mathrm{L}$ ) was added and the cells were incubated for an additional $48 \mathrm{~h}$. Then, the cells were fixed using $3.3 \%(w / v)$ trichloroacetic acid at $4{ }^{\circ} \mathrm{C}$ for $1 \mathrm{~h}$ and stained with $0.4 \%$ SRB dissolved in $1 \%(v / v)$ acetic acid for $30 \mathrm{~min}$. Unbound dye was removed by four washes with $1 \%$ acetic acid, and protein-bound dye was extracted with $10 \mathrm{mmol} / \mathrm{L}$ Tris base ( $\mathrm{pH}$ 10.5) for $5 \mathrm{~min}$. The optical density was determined at $510 \mathrm{~nm}$ using a VersaMax Microplate Reader (Molecular Devices). The experiments were performed in triplicate. The $\mathrm{IC}_{50}$ values were derived from a nonlinear regression model (curvefit) based on a sigmoidal dose response curve (variable slope) and computed using GraphPad Prism (Version 5.02, GraphPad Software Inc, San Diego, CA, USA). The data were expressed as the mean \pm SEM.

\section{Results}

Physalin B induces apoptosis and an initial stage of autophagy in human HCT116 colon cancer cells

The anticancer activity of physalin B (Figure 1A) was evaluated in HCT116 colon cancer cells using the MTT viability assay. Nuclei staining and Western blot analysis were utilized to detect the apoptotic status of HCT116 cells treated with physalin B. Physalin B exerted anticancer activity with an $\mathrm{IC}_{50}$ value of approximately $1.35 \mu \mathrm{mol} / \mathrm{L}$ (Figure 1B). Physalin $B$ induced pyknosis and the formation of apoptotic bodies (Figure 1C), PARP cleavage and caspase-3 cleavage (Figure 1D) after a $24 \mathrm{~h}$ treatment, suggesting that physalin B induced apoptosis in HCT116 cells.

Others have reported that physalin B could inhibit UPP, but its effect on ALP has not been studied. Here we used GFPLC3 stably-transfected HCT116 cells to monitor autophagosome formation. After a $12 \mathrm{~h}$ treatment, physalin B induced autophagosome formation in GFP-LC3 stably-transfected HCT116 cells (Figure 1E). In addition, Western blot detection of autophagy marker LC3-II showed the accumulation of LC3II in a time- and dose-dependent manner (Figure 1F), suggesting that autophagy was occurring.

\section{Beclin 1 and mTOR are not required for the physalin B-induced} autophagic response

Beclin 1 is a canonical autophagy marker that has a central role in autophagy. The expression level of Beclin 1 is upregulated during the canonical autophagic process. Autophagy can also be induced by the inhibition of TOR (target of rapamycin). $\mathrm{mTOR}$, a serine/threonine protein kinase that balances diverse anabolic processes such as cell proliferation, cell survival, and protein synthesis, plays a vital role at the interface of the pathways that coordinately regulate the balance between cell growth and autophagy. Here we detected the protein level of Beclin 1 and phosphorylation level of mTOR at Ser-2448 using Western blot analysis. As shown in Figure 1F, after a $9 \mathrm{~h}$ treatment, physalin B induced LC3-II accumulation, indicating the initiation of autophagosome formation. Additionally, the protein level of Beclin 1 decreased and the phosphorylation level of mTOR did not change, suggesting that Beclin1 and $\mathrm{mTOR}$ are not required for the physalin B-induced autophagic response. 
A

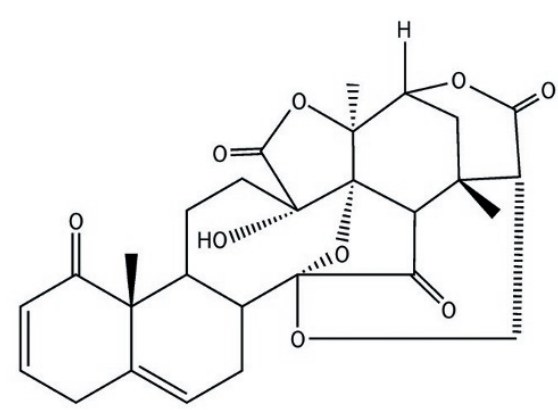

C

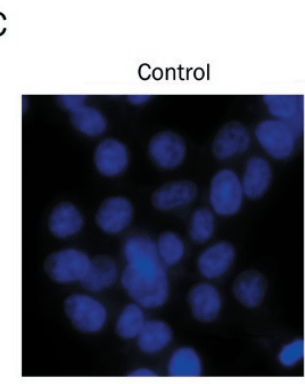

E

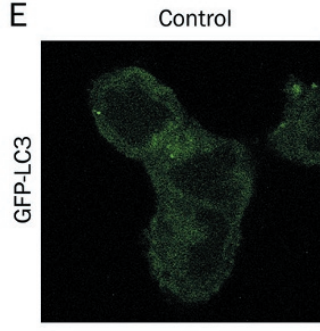

F

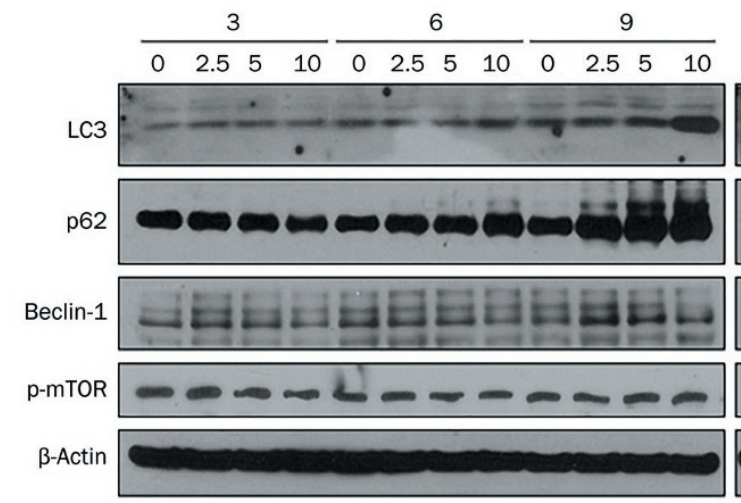

B

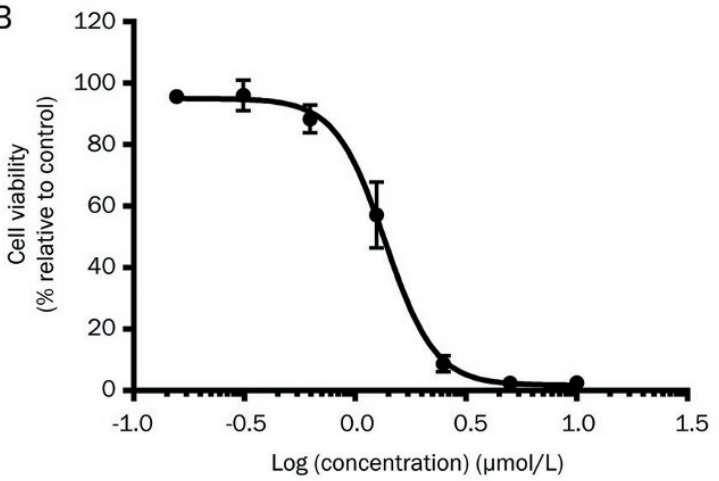

D

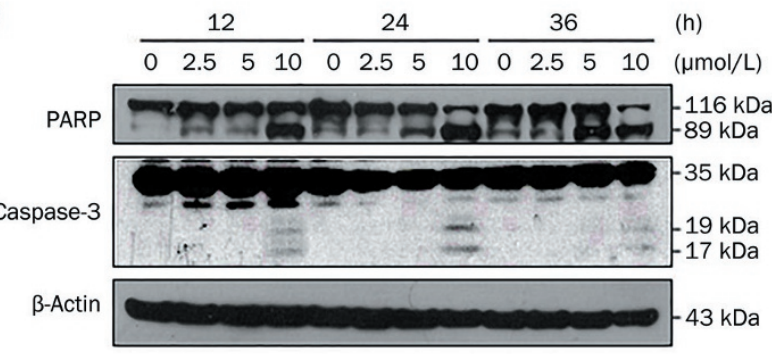

Figure 1. Physalin B induced apoptosis and an initial stage of autophagy in HCT116 colon cancer cells. (A) The chemical structure of physalin B. (B) Physalin B inhibited HCT116 cell viability. Cells were treated with physalin B at doses ranging from $10 \mu \mathrm{mol} / \mathrm{L}$ to $0.31 \mu \mathrm{mol} / \mathrm{L}$ for $72 \mathrm{~h}$. The cell viability was assessed by MTT assay. $\mathrm{IC}_{50}$ value is expressed as the mean $\pm S E M$ of four independent experiments. (C) Physalin B induced pyknosis and formation of apoptotic bodies. Cells were treated with 5 and $10 \mu \mathrm{mol} / \mathrm{L}$ physalin B for $24 \mathrm{~h}$ and further stained with Hoechst 33342 and observed under microscope. (D) Cells were treated with $2.5,5$, and $10 \mu \mathrm{mol} / \mathrm{L}$ physalin B. Samples were collected at 12,24 , and $36 \mathrm{~h}$, separately. Levels of PARP and caspase-3 were detected using Western blotting assay. (E) Physalin B induced autophagosome formation in GFP-LC3-expressing HCT116 cells. GFP-LC3-expressing HCT116 cells were treated with $10 \mu \mathrm{mol} / \mathrm{L}$ physalin B, 0.1\% DMSO (negative control) and $5 \mu \mathrm{mol} / \mathrm{L}$ rapamycin (positive control) for $12 \mathrm{~h}$. Autophagosomes were observed under an Olympus Fluoview FV1000 confocal microscope (Olympus, Tokyo, Japan). (F) Cells were treated with 2.5, 5, and 10 mol/L physalin B. Samples were collected at 3, 6, 9, 12, 24, and 36 h separately. Levels of LC3, p62, Beclin-1, pSer2448mTOR, and $\beta$-actin were detected using Western blotting assay. 
Physalin B impairs the fusion of autophagosomes with lysosomes in the late stage of autophagy

p62 accumulates when autophagy is inhibited, and decreased levels can be observed when autophagy is induced. Thus, p62 may be used as a marker to study autophagic flux. Here, p62 levels accumulated within $36 \mathrm{~h}$ after treatment, suggesting an inhibition of autophagic degradation (Figure 1F).

Once formed, autophagosomes must reach lysosomes to fuse with them for the degradation of the contents ${ }^{[26]}$. Here, the localization of autophagosomes and lysosomes was further monitored in GFP-LC3 (autophagosome marker) and RFP-LAMP1 (lysosomal marker) co-transfected cells. The colocalization of autophagosomes and lysosomes was blocked in physalin B-treated cells as shown in Figure 2A. As reported, microtubules appear to be essential for the delivery of autophagosomes to lysosomes for autophagosome/lysosome fusion $^{[27]}$. In addition, the F-actin network has also been found to stimulate autophagosome/lysosome fusion and substrate degradation $^{[28]}$. We also evaluated the status of microtubules and F-actin. As shown in Figure 2B and 2C, microtubules and F-actin microfilaments were altered, which may be involved in the blockage of autophagosome/lysosome fusion and conse- quent incomplete autophagic degradation.

Mitogen-activated protein kinase (MAPK) pathway regulates physalin B-induced autophagy and apoptosis

Western blot analysis was used to detect the phosphorylation levels of ERK1/2, JNK, and p38 MAPK. As shown in Figure $3 \mathrm{~A}$, the phosphorylation levels of ERK1/2, JNK, and p38 MAPK were increased in a time- and dose- dependent manner, indicating a sustained activation of the ERK1/2, JNK, and p38 MAPK pathways. In addition, the p38 MAPK inhibitor SB202190, ERK inhibitor U0126, and JNK inhibitor SP600125 partially reversed PARP cleavage and p62 accumulation (Figure 3B), suggesting that the ERK, p38, and JNK pathways participated in physalin B-induced apoptosis and autophagy.

\section{Physalin B induces mito-ROS production}

Previous studies have reported that mitochondrial ROS plays an important role in autophagy ${ }^{[29]}$. MitoSOX staining was performed to detect whether physalin B could induce mito-ROS. As Figure 4 shows, physalin B induced mito-ROS generation in a dose-dependent manner as early as $4 \mathrm{~h}$ after treatment. The ROS scavenger NAC effectively reversed the generation
A
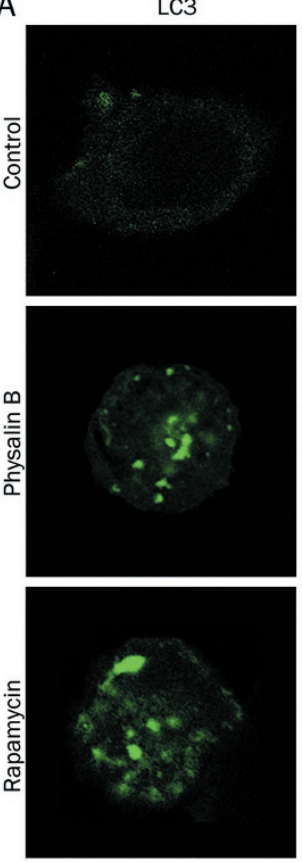

LAMP1
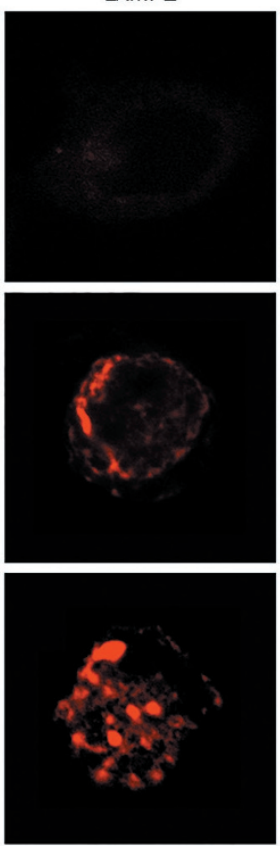

Merge
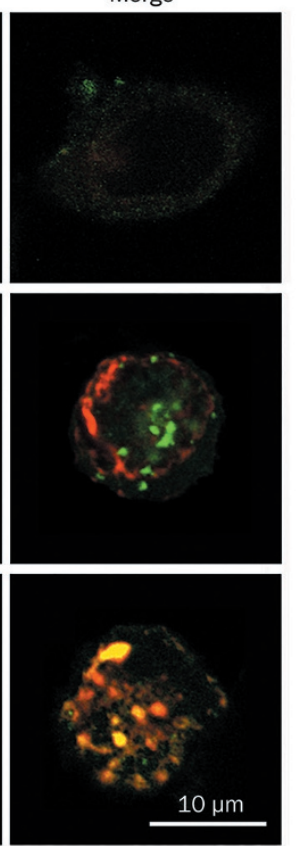

B
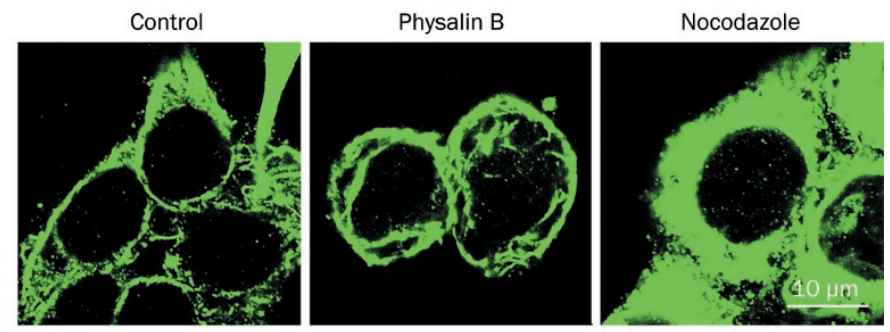

C

Control

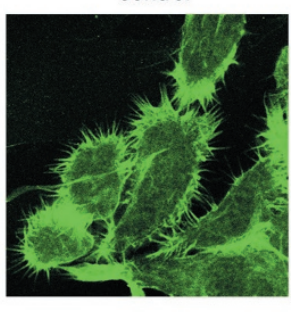

Physalin B

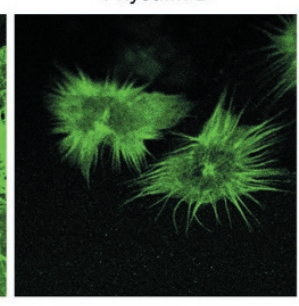

Rapamycin

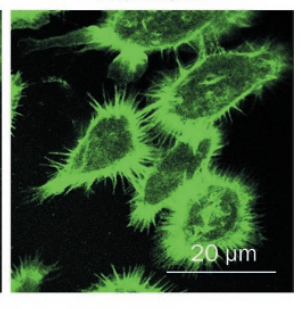

Figure 2. Autophagosomes were not co-localized with lysosomes in physalin B-treated cells. (A) Autophagosomes were not co-localized with lysosomes. GFP-LC3/RFP-LAMP1-co-transfected cells were treated with $10 \mu \mathrm{mol} / \mathrm{L}$ physalin B, 0.1\% DMSO (negative control) and $5 \mu \mathrm{mol} / \mathrm{L}$ rapamycin (positive control) for $12 \mathrm{~h}$. Localization of GFP-LC3 and RFP-LAMP1 were observed under an Olympus Fluoview FV1000 confocal microscope (Olympus, Tokyo, Japan). (B) Microtubule cytoskeleton was changed in physalin B-treated cells. Cells were treated with $10 \mu \mathrm{mol} / \mathrm{L}$ physalin B, 0.1\% DMSO (negative control) and $100 \mathrm{ng} / \mathrm{mL}$ nocodazole (positive control) for $12 \mathrm{~h}$. Samples were then prepared as mentioned in the "Materials and methods" section, and the status of microtubules was observed using an Olympus confocal microscope (Olympus, Tokyo, Japan). (C) F-actin microfilaments were changed in physalin B-treated cells. Cells were treated with $10 \mu \mathrm{mol} / \mathrm{L}$ physalin B, 0.1\% DMSO (negative control) and $5 \mu \mathrm{mol} / \mathrm{L}$ rapamycin for $12 \mathrm{~h}$. Samples were then prepared as mentioned in the "Materials and methods" section, and the status of F-actin microfilaments was observed using an Olympus confocal microscope (Olympus, Tokyo, Japan). 
A

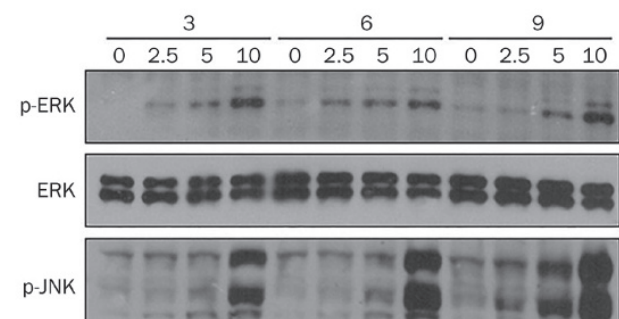

JNK

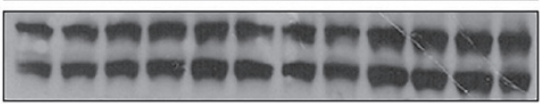

p-p38

p38
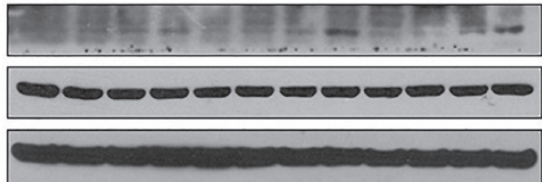

B-Actin

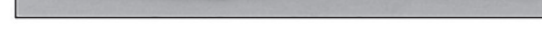

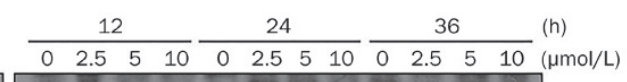
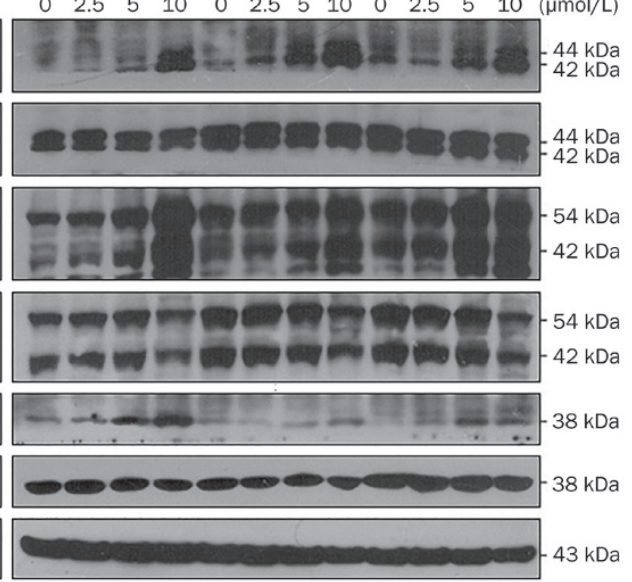

B

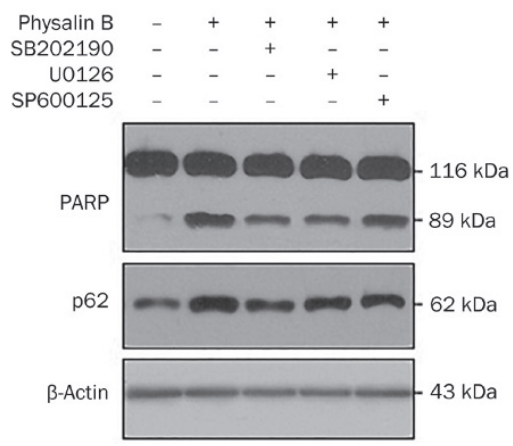

Figure 3. ERK, JNK, and p38 MAPK were activated in physalin B-treated cells. (A) Cells were treated with 2.5, 5, and $10 \mu \mathrm{mol} / \mathrm{L}$ physalin B. Samples were collected at 3, 6, 9, 12, 24, and $36 \mathrm{~h}$, separately. Levels of phosphor-ERK, ERK, phosphor-JNK, JNK, phosphor-p38 MAPK, p38 MAPK, and $\beta$-actin were detected using Western blotting assay. (B) Cells were treated with $10 \mu \mathrm{mol} / \mathrm{L}$ physalin B with or without p38 MAPK inhibitor SB202190, ERK inhibitor U0126, and JNK inhibitor SP600125. Levels of PARP, p62, and $\beta$-actin were detected using Western blotting assay.

of ROS (Figure 4).

\section{ROS scavenger NAC reverses UPP inhibition and incomplete} autophagic response

To evaluate the role of mito-ROS generation in the anticancer effect of physalin B, NAC was used to eliminate mito-ROS in physalin B-treated cells. The effect of mito-ROS on UPP inhibition, autophagosome formation, cytoskeletal changes, the MAPK pathways, apoptosis, and cellular viability was evaluated. Here, NAC treatment effectively reduced physalin B-induced mito-ROS (Figure 4). The elimination of ROS upon NAC treatment could abrogate UPP inhibition as the polyubiquitin level was significantly downregulated (Figure 5A). Mito-ROS elimination reversed the incomplete autophagic response as demonstrated by the increased colocalization of autophagosomes and lysosomes (Figure 5B) and decreased p62 levels (Figure 5E). Changes in microtubules and F-actin microfilaments were also reversed upon NAC treatment, suggesting that autophagosome/lysosome fusion process was recovered (Figure 5C and 5D). We also investigated the role of ROS in MAPK pathway activation, which has a close relationship with apoptosis and autophagy. As shown in Figure $5 \mathrm{E}$, the phosphorylation levels of ERK, p38, and JNK were reduced effectively upon NAC treatment, indicating that MAPK pathways were activated via ROS generation. Apoptosis and impaired viability induced by physalin $\mathrm{B}$ can also be reversed upon ROS elimination (Figure 5F-5H), indicating that the anticancer activity of physalin $\mathrm{B}$ relied on ROS generation.

\section{Discussion}

The UPP is a major degradation system for short-lived proteins ${ }^{[30]}$. Proteins destined for degradation through the UPP are labeled with ubiquitin and then degraded by the $26 \mathrm{~S}$ proteasome complex. Precise elimination of these proteins is critical to the accurate regulation of intracellular signaling pathways involved in multiple cellular processes, such as cell proliferation and cell death. 


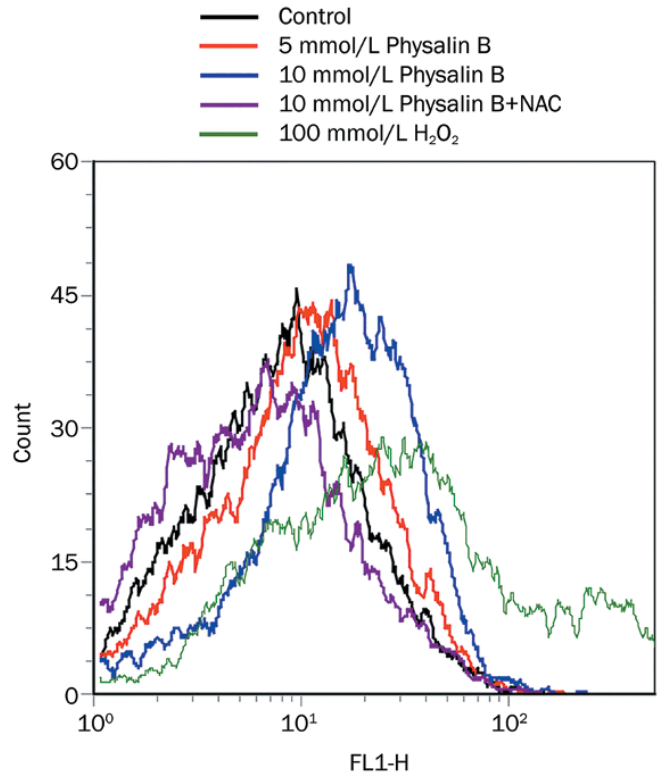

Figure 4. Physalin B induced mitochondrial ROS generation. Cells were treated for $4 \mathrm{~h}$ with physalin $\mathrm{B}(5$ and $10 \mu \mathrm{mol} / \mathrm{L})$, physalin $\mathrm{B}(10 \mu \mathrm{mol} / \mathrm{L})$ with NAC (10 mmol/L), DMSO (0.1\%) as negative control, $\mathrm{H}_{2} \mathrm{O}_{2}(100 \mu \mathrm{mol} / \mathrm{L})$ as positive control. Level of mitochondrial ROS was detected by MitoSox ${ }^{\mathrm{TM}}$ Red mitochondrial superoxide indicator according to the manufacturer's instructions.

The ALP is another major intracellular degradation system, which is mainly responsible for the degradation of long-lived proteins and other cellular contents ${ }^{[31]}$. Autophagy is stimulated by multiple signals, including nutrient deprivation, oxidative stress, hypoxia, DNA damage, and endoplasmic reticulum stress. This dynamic process involves autophagosome formation and fusion with lysosomes, followed by lysosomal degradation of the intra-autophagosomal contents.

Increasing evidence has demonstrated the crosstalk between the UPP and ALP. For example, upon the inhibition of the UPP, cells activate ALP to facilitate autophagolysosomal protein turnover ${ }^{[14]}$. Additionally, ALP inhibition increases the levels of proteasome substrates via excess p62/SQSTM1 accumulation, which leads to an inhibition of the clearance of ubiquitinated proteins designated for proteasomal degradation by delaying their delivery to the proteases of the proteasome ${ }^{[32]}$. The accumulation of ubiquitinated proteins is toxic and can induce apoptosis. Here, physalin B inhibited the UPP and ALP, followed by the accumulation of ubiquitinated proteins, thus accelerating apoptotic cell death.

The natural compound physalin $B$ has been reported as an indirect UPP inhibitor ${ }^{[13]}$. The effect of physalin B on the ALP was investigated here. LC3 exists in two forms, LC3-I and its proteolytic derivative LC3-II. LC3-I is localized in the cytoplasm and LC3-II is localized in autophagosomal membranes. Upon the initiation of autophagy, the C-terminal glycine of LC3-I is conjugated to phosphatidylethanolamine to form LC3II, which is targeted rapidly to autophagic membranes. Similarly, the GFP-LC3 fusion protein, which is in a diffuse pattern (cytoplasmic and nuclear), redistributes to a vacuolar pattern when autophagic vacuoles are formed ${ }^{[33]}$. The abundance of LC3-II is closely related with autophagosome quantity and can be used as a reliable indicator of autophagosome presence ${ }^{[34]}$. p62/SQSTM1 is an ubiquitin-binding scaffold protein that is degraded by autophagy and may be probed as a marker for the induction of autophagy, clearance of protein aggregates, and the inhibition of autophagy ${ }^{[35]}$. Physalin B induced the formation of autophagosomes and upregulation of LC3-II, indicating the initiation of autophagy. Beclin 1, the mammalian ortholog of yeast Atg6/Vps30, is a canonical autophagy marker that has a central role in canonical autophagy. During the canonical autophagic process, Beclin 1 is important for the localization of autophagic proteins to a pre-autophagosomal structure $^{[36]}$. The expression level of Beclin 1 is upregulated during canonical autophagy ${ }^{[37-39]}$. When apoptosis occurs and caspase-3 is activated, Beclin 1 is cleaved by caspase-3, generating fragments that lose their ability to induce autophagy ${ }^{[40]}$. Here, non-canonical Beclin 1-independent autophagy was initiated in physalin B-treated cells. After treatment with physalin B for $9 \mathrm{~h}$, the protein levels of LC3-II and p62 increased, whereas the protein level of Beclin 1 was not upregulated compared with the control group, indicating that the initiation of the autophagy response was not dependent on Beclin 1. After a $12 \mathrm{~h}$ treatment, caspase- 3 was activated and the expression level of Beclin 1 was reduced, suggesting that Beclin 1 was cleaved by caspase- 3 and its pro-autophagic activity was lost.

The ALP can also be induced by nutrient starvation in eukaryotic cells, which is largely due to the inhibition of TOR (target of rapamycin) ${ }^{[41]}$. mTOR, a serine/threonine protein kinase that balances diverse anabolic processes such as cell proliferation, cell survival, and protein synthesis, plays a vital role at the interface of the pathways that coordinately regulate the balance between cell growth and autophagy ${ }^{[42]}$. Here, autophagosomes were observed after a $12 \mathrm{~h}$ treatment without a reduction in the phosphorylation of mTOR at the serine 2448 , a key determinant of mTOR activity ${ }^{[33]}$, suggesting that the initiation of autophagy is mTOR-independent.

Further investigation showed a blockage during the late degradation stage of autophagy in physalin B-treated cells. LC3-II and p62, which should be degraded through the fusion of autophagosomes with lysosomes during the late stage of autophagy, were not degraded but rather accumulated within a $36 \mathrm{~h}$ treatment. This result implied incomplete lysosomal degradation. Additional observation of autophagosomes and lysosomes in GFP-LC3/RFP-LAMP1 transfected cells indicated a blockage of the co-localization and fusion of lysosomes and autophagosomes during late stage of autophagy.

The cytoskeleton is a network of protein polymer fibrils that maintains cell shape, compartmentalization and intracellular trafficking or even whole-cell movement ${ }^{[44]}$. Microfilaments, microtubules, and intermediate filaments combine to constitute the cytoskeletal system. Microtubule and microfilament systems are tightly regulated to facilitate a dynamic organization and rapid remodeling of the cytoskeleton, and they play 
A

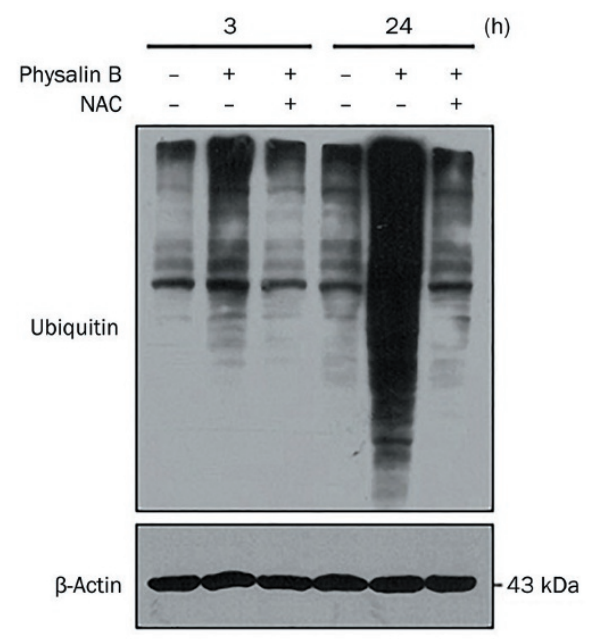

B
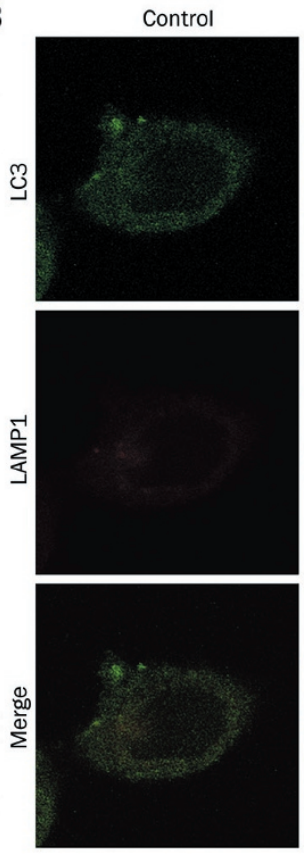

Physalin B
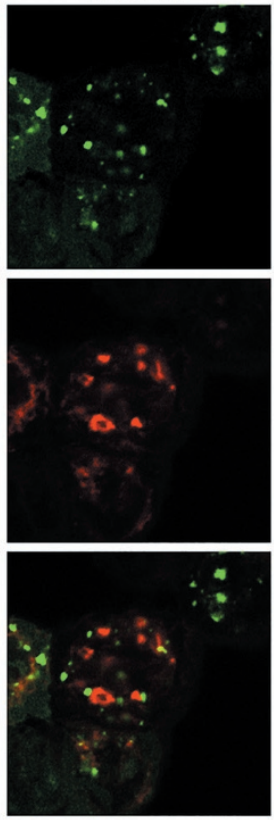

Physalin B+NAC
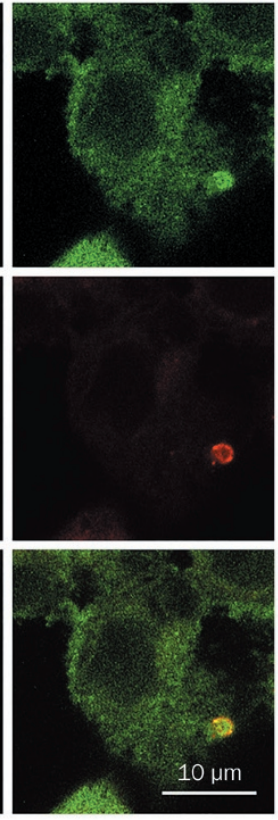

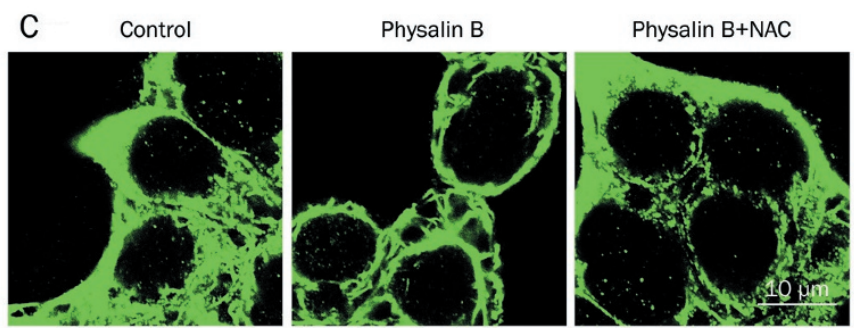

E

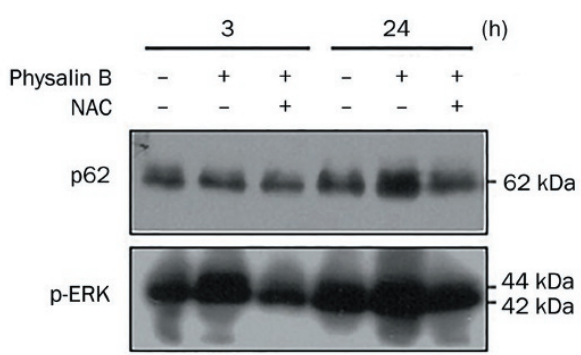

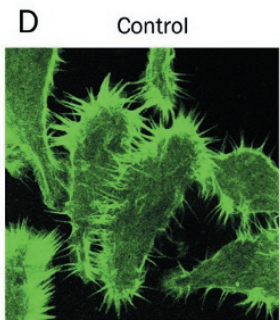

F

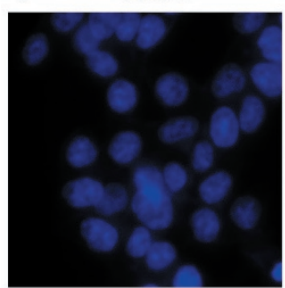

Physalin B

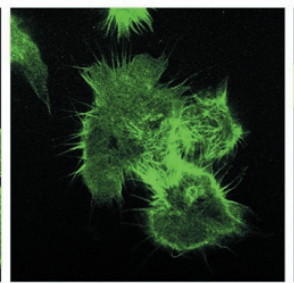

Physalin B

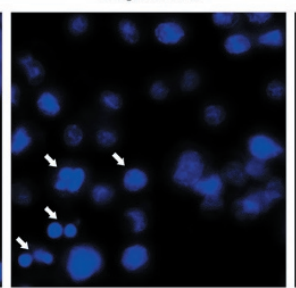

Physalin B+NAC

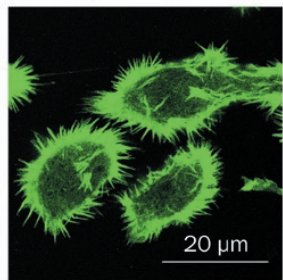

Physalin B+NAC

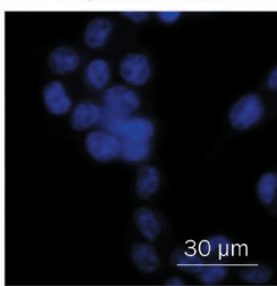

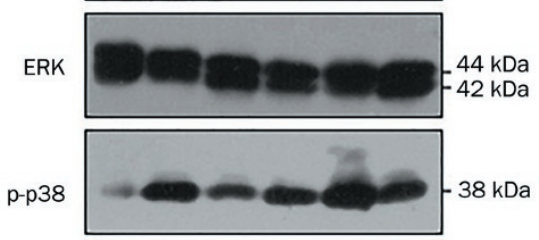
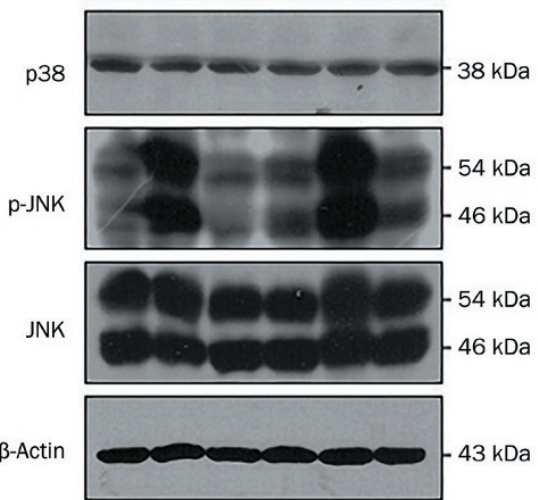

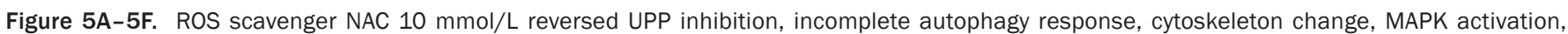

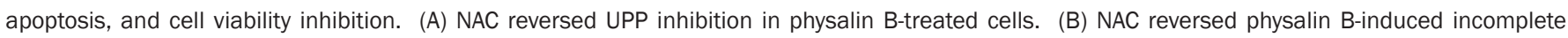

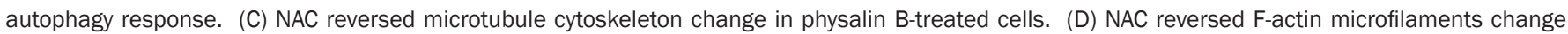

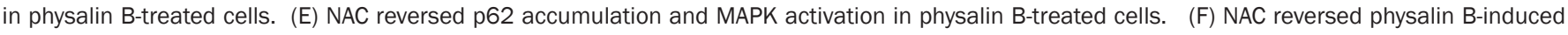
pyknosis and formation of apoptotic bodies. 
G

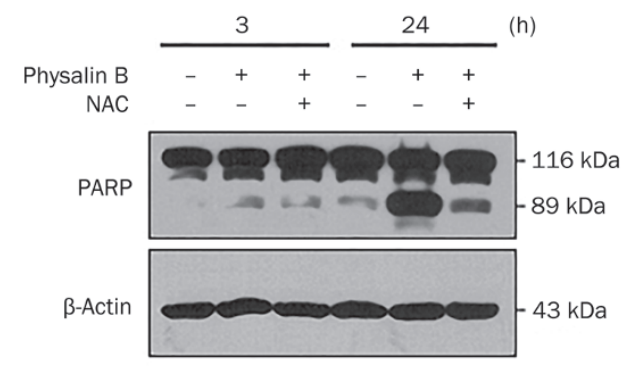

$\mathrm{H}$

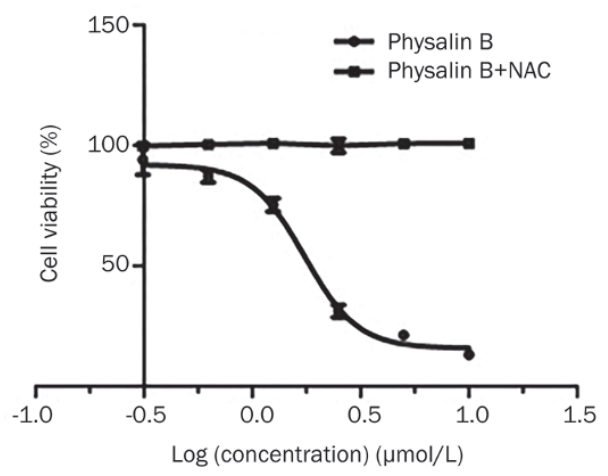

Figure 5G-5H. (G) NAC reversed physalin B-induced PARP cleavage. (H) NAC reversed cell viability reduction in physalin B-treated cells. IC ${ }_{50}$ value is expressed as the mean \pm SEM of four independent experiments.

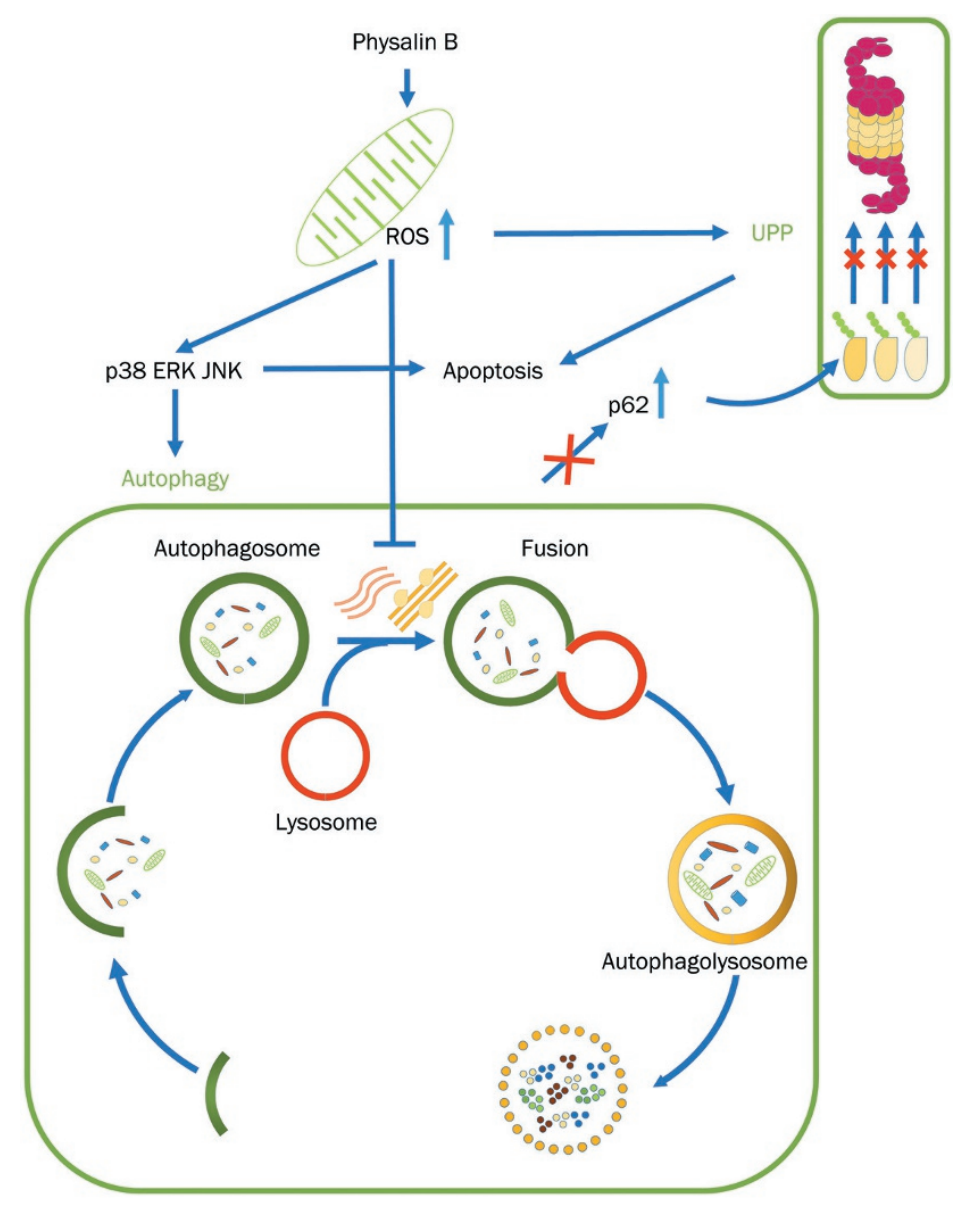

Figure 6. A schematic model of the anticancer mechanism of physalin B in HCT116 cells. Physalin B induces mito-ROS generation resulting in UPP inhibition and incomplete non-canonical autophagy response. Autophagosome/lysosome fusion is blocked due to the inhibition of functions of microtubules and F-actin microfilaments, leading to autophagy substrates accumulation which accelerates apoptosis. Besides, MAPK pathways are sustainedly activated and partially mediate autophagy response and apoptosis in physalin B-treated cells.

an important role in specific aspects of autophagy ${ }^{[44]}$. Microtubules facilitate autophagosome trafficking. Intact microtubules contribute to the fusion of autophagosomes with lysosomes, which could be disrupted by microtubule-depolymerizing reagents such as nocodazole. In addition, microfilaments are also found to facilitate autophagosome/lysosome fusion and substrate degradation ${ }^{[45]}$. Here, the status of microtubules and F-actin microfilaments were altered, a possible mechanism 
that might explain the blockage of autophagosome/lysosome fusion and substrate degradation.

Interestingly, He et al found that physalin A induced Beclin 1-dependent autophagy, which protected human melanoma A375-S2 cells from apoptosis ${ }^{[46]}$. Unlike physalin A, physalin $\mathrm{B}$ induced a Beclin 1-independent autophagic response. As previously reported, ROS-induced activation of ERK and JNK simultaneously induce non-canonical autophagy and apoptosis in cancer cells ${ }^{[47]}$. Here, ERK, JNK, and p38 MAPK were activated via ROS generation. The inhibition of ERK, JNK, and p38 MAPK, separately, partially reversed PARP cleavage and p62 accumulation, indicating that the MAPK pathways partially mediated physalin B-induced apoptosis and a noncanonical autophagic response.

In addition, an increase of mito-ROS was observed. As reported, ROS could impact many different cellular processes ${ }^{[48]}$, including the inhibition of the UPP ${ }^{[49]}$, induction of the $\mathrm{ALP}^{[50]}$, cytoskeleton rearrangement ${ }^{[51]}$, and activation of the MAPK pathways ${ }^{[52]}$.

The ROS scavenger NAC effectively reversed ubiquitinated protein accumulation, autophagosome formation, and cytoskeletal network changes, indicating that UPP inhibition and the incomplete autophagic response depended on ROS generation. NAC treatment also reversed MAPK pathway activation, apoptosis and cell viability inhibition.

In conclusion, our findings reveal the antitumor mechanism of physalin B in HCT116 cells: mito-ROS induction by physalin B results in UPP inhibition and an incomplete non-canonical autophagy response. The incompletion of autophagic degradation might be due to changes in the microtubules and F-actin network, followed by a blockage of autophagosome/ lysosome fusion. Physalin B induces sustained activation of the ERK, JNK, and p38 MAPK pathways, which partially mediates an incomplete autophagic response and apoptosis (Figure 6).

Here we report that physalin B is not only a UPP inhibitor but also an inducer of an incomplete autophagic response via mito-ROS generation. Because the UPP and ALP are two important potential targets for anticancer therapy, the discovery and mechanistic study of the natural compound physalin $B$ with a novel mode of action provides important knowledge for potent anticancer drug discovery and development.

\section{Abbreviations}

UPP, ubiquitin-proteasome pathway; ALP, autophagylysosome pathway; GFP, green fluorescent protein; RFP, red fluorescent protein; LC3, microtubule-associated protein 1-light chain 3; MAPK, mitogen-activated protein kinase; SRB, Sulforhodamine B; NAC, N-Acetyl-L-Cysteine; ROS, reactive oxygen species; ATCC, American type cell collection; DMSO, dimethyl sulfoxide; FBS, fetal bovine serum.

\section{Acknowledgements}

This work was supported by grants from the National Natural Science Foundation of China (No 81473244, 81270942, and 81125023), and the National Science and Technology Major
Projects for "Major New Drugs Innovation and Development" (2012ZX09301001-004, 2013ZX09508104).

\section{Author contribution}

Yi-ming MA, Yu-bo ZHOU, Li-hong HU, and Jia LI designed the research; Yi-ming MA and Wei HAN performed the research; Yi-ming MA, Yu-bo ZHOU, Li-hong HU, and Jia LI analyzed the data; Yi-ming MA and Yu-bo ZHOU wrote the paper.

\section{References}

1 Adriana BR, Rafael ML, Gilberto S. Natural products in anticancer therapy. Curr Opin Pharmacol 2001; 1: 364-9.

2 Claudia C, Flavia R, Mario D, Marc D. Natural compounds as regulators of the cancer cell metabolism. Int J Cell Biol 2013. doi:10.1155/2013/639401.

3 Slichenmyer WJ, Von Hoff DD. Taxol: a new and effective anti-cancer drug. Anticancer Drugs 1991; 2: 519-30.

4 Liu G, Ma YM, Tai WY, Xie CM, Li YL, Li J, et al. Design, synthesis, and biological evaluation of caprolactam-modified bengamide analogues. ChemMedChem 2008; 3: 74-8.

5 Tai WY, Zhang RT, Ma YM, Gu M, Liu G, Li J, et al. Design, synthesis, and biological evaluation of ring-opened bengamide analogues. ChemMedChem 2011; 6: 1555-8.

6 Hande KR. Etoposide: four decades of development of a topoisomerase II inhibitor. Eur J Cancer 1998; 34: 1514-21.

$7 \mathrm{Xu}$ Y, Villalona-Calero MA. Irinotecan: mechanisms of tumor resistance and novel strategies. Ann Oncol 2002; 13: 1841-51.

8 Jordan MA, Hirnes RH, Wilson L. Comparison of the effects of vinblastine, vincristine, vindesine, and vinepidine on microtubule dynamics and cell proliferation in vitro. Cancer Res 1985; 45: 27417.

9 Lao J, Madani J, Puértolas T, Álvarez M, Hernández A, Pazo-Cid R, et al. Liposomal doxorubicin in the treatment of breast cancer patients: a review. J Drug Deliv 2013; 2013: Article ID 456409, 12 pages. doi:10.1155/2013/456409.

10 Bolwell BJ, Cassileth PA, Gale RP. High dose cytarabine: a review. Leukemia 1988; 2: 253-60.

11 Santos RA, Cabral TR, Cabral IR, Antunes LM, Andrade CP, Cardoso PCS, et al. Genotoxic effect of Physalis angulata L (Solanaceae) extract on human lymphocytes treated in vitro. Biocell 2008; 32 : 195-200.

12 Magalhães HI, Veras ML, Torres MR, Alves AP, Pessoa OD, Silveira ER, et al. In-vitro and in-vivo antitumour activity of physalins $B$ and $D$ from Physalis angulata. J Pharm Pharmacol 2006; 58: 235-41.

13 Vandenberghe I, Créancier L, Vispé S, Annereau JP, Barret JM, Pouny I, et al. Physalin B, a novel inhibitor of the ubiquitin-proteasome pathway, triggers NOXA-associated apoptosis. Biochem Pharmacol 2008; 76: 453-62.

14 Zhu K, Dunner K Jr, McConkey DJ. Proteasome inhibitors activate autophagy as a cytoprotective response in human prostate cancer cells. Oncogene 2010; 29: 451-62.

15 Ding WX, Ni HM, Gao WT, Yoshimori T, Stolz DB, Ron D, et al. Linking of autophagy to ubiquitin-proteasome system is important for the regulation of endoplasmic reticulum stress and cell viability. Am J Pathol 2007; 171: 513-24.

16 Teicher BA, Ara G, Herbst R, Palombella VJ, Adams J. The proteasome inhibitor PS-341 in cancer therapy. Clin Cancer Res 1999; 5: 263845

17 Sterz J, von Metzler I, Hahne JC, Lamottke B, Rademacher J, Heider U, 
et al. The potential of proteasome inhibitors in cancer therapy. Expert Opin Investig Drugs 2008; 17: 879-95.

18 Cheong $\mathrm{H}$, Lu C, Lindsten T, Thompson CB. Therapeutic targets in cancer cell metabolism and autophagy. Nat Biotechnol 2012; 30: 671-8.

19 Yao TP. The role of ubiquitin in autophagy-dependent protein aggregate processing. Genes Cancer 2010; 1: 779-86.

20 Ge PF, Zhang JZ, Wang XF, Meng FK, Li WC, Luan YX, et al. Inhibition of autophagy induced by proteasome inhibition increases cell death in human SHG-44 glioma cells. Acta Pharmacol Sin 2009; 30: 104652.

21 Ma L, Ali M, Arfan M, Lou LG, Hu LH. Withaphysanolide A, a novel C-27 norwithanolide skeleton, and other cytotoxic compounds from Physalis divericata. Tetrahedron Lett 2007; 48: 449-52.

22 Matsuura T, Kawai M, Makashima R, Butsugan Y. Structures of physalin A and physalin B, 13,14-seco-16,24-cyclo-steroids from Physalis alkekengi var. Francheti. J Chem Soc Perkin 1 1970; 5: 66470.

23 Ma YM, Zhou YB, Xie CM, Chen DM, Li J. Novel microtubule-targeted agent 6-chloro-4-(methoxyphenyl) coumarin induces $\mathrm{G}_{2}-\mathrm{M}$ arrest and apoptosis in HeLa cells. Acta Pharmacol Sin 2012; 33: 407-17.

24 Zang Y, Yu LF, Nan FJ, Feng LY, Li J. AMP-activated protein kinase is involved in neural stem cell growth suppression and cell cycle arrest by 5 -aminoimidazole-4-carboxamide-1- $\beta$-d-ribofuranoside and glucose deprivation by down-regulating phospho-retinoblastoma protein and cyclin D. J Biol Chem 2009; 284: 6175-84.

25 Zang Y, Yu LF, Pang T, Fang LP, Feng X, Wen TQ, et al. AICAR induces astroglial differentiation of neural stem cells via activating the JAK/ STAT3 pathway independently of AMP-activated protein kinase. J Biol Chem 2008; 283: 6201-8.

26 Jahreiss L, Menzies FM, Rubinsztein DC. The itinerary of autophagosomes: from peripheral formation to kiss-and-run fusion with lysosomes. Traffic 2008; 9: 574-87.

27 Webb JL, Ravikumar B, Rubinsztein DC. Microtubule disruption inhibits autophagosome-lysosome fusion: implications for studying the roles of aggresomes in polyglutamine diseases. Int J Biochem Cell Biol 2004; 36: 2541-50.

28 Lee JY, Koga H, Kawaguchi Y, Tang W, Wong E, Gao YS, et al. HDAC6 controls autophagosome maturation essential for ubiquitin-selective quality-control autophagy. EMBO J 2010; 29: 969-80.

29 Scherz-Shouval R, Elazar Z. ROS, mitochondria and the regulation of autophagy. Trends Cell Biol 2007; 17: 422-7.

30 Zolk 0, Schenke C, Sarikas A. The ubiquitin-proteasome system: focus on the heart. Cardiovasc Res 2006; 70: 410-21.

31 Yang Z, Klionsky DJ. Eaten alive: a history of macroautophagy. Nat Cell Biol 2010; 12: 814-22.

32 Thannickal VJ, Fanburg BL. Reactive oxygen species in cell signaling. Am J Physiol Lung Cell Mol Physiol 2000; 279: L1005-28.

33 Boya P, González-Polo RA, Casares N, Perfettini JL, Dessen P, Larochette $\mathrm{N}$, et al. Inhibition of macroautophagy triggers apoptosis. Mol Cell Biol 2005; 25: 1025-40.

34 Mizushima N, Yoshimorim T, Levine B. Methods in mammalian autophagy research. Cell 2010; 140: 313-26.

35 Bjørkøy G. Monitoring autophagic degradation of p62/SQSTM1.
Methods Enzymol 2009; 452: 181-97.

36 Kang R, Zeh HJ, Lotze MT, Tang D. The Beclin 1 network regulates autophagy and apoptosis. Cell Death Differ 2011; 18: 571-80.

37 Chang KH, Yan MD, Yao CJ, Lin PC, Lai GM. Honokiol-induced apoptosis and autophagy in glioblastoma multiforme cells. Oncol Lett 2013; 6: 1435-8.

38 Can G, Ekiz HA, Baran Y. Imatinib induces autophagy through BECLIN-1 and ATG5 genes in chronic myeloid leukemia cells. Hematology 2011; 16: 95-9.

39 Dong Q, Oh JE, Yi JK, Kim RH, Shin KH, Mitsuyasu R, et al. Efavirenz induces autophagy and aberrant differentiation in normal human keratinocytes. Int J Mol Med 2013; 31: 1305-12.

40 Djavaheri-Mergny M, Maiuri MC, Kroemer G. Cross talk between apoptosis and autophagy by caspase-mediated cleavage of Beclin 1. Oncogene 2010; 29: 1717-9.

41 Russell RC, Yuan HX, Guan KL. Autophagy regulation by nutrient signaling. Cell Res 2014; 24: 42-57.

42 Jung $\mathrm{CH}$, Ro SH, Cao J, Otto NM, Kim DH. mTOR regulation of autophagy. FEBS Lett 2010; 584: 1287-95.

43 Sukumari-Ramesh S, Singh N, Dhandapani KM, Vender JR. mTOR inhibition reduces cellular proliferation and sensitizes pituitary adenoma cells to ionizing radiation. Surg Neurol Int 2011; 2: 22.

44 Monastyrska I, Rieter E, Klionsky DJ, Reggiori F. Multiple roles of the cytoskeleton in autophagy. Biol Rev 2009; 84: 431-48.

45 Lee JY, Koga H, Kawaguchi Y, Tang W, Wong E, Gao YS, et al. HDAC6 controls autophagosome maturation essential for ubiquitin-selective quality-control autophagy. EMBO J 2010; 29: 969-80.

$46 \mathrm{He} \mathrm{H}$, Zang LH, Feng YS, Chen LX, Kang N, Tashiro S, et al. Physalin A induces apoptosis via p53-Noxa-mediated ROS generation, and autophagy plays a protective role against apoptosis through p38-NFKB survival pathway in A375-S2 cells. J Ethnopharmacol 2013; 148: 544-55.

47 Wong $\mathrm{CH}$, Iskandar KB, Yadav SK, Hirpara JL, Loh T, Pervaiz S. Simultaneous induction of non-canonical autophagy and apoptosis in cancer cells by ROS-dependent ERK and JNK activation. PloS One 2010. doi: 10.1371/ journal.pone.0009996.

48 Thannickal VJ, Fanburg BL. Reactive oxygen species in cell signaling. Am J Physiol Lung Cell Mol Physiol 2000; 279: L1005-28.

49 Aiken CT, Kaake RM, Wang XR, Huang L. Oxidative stress-mediated regulation of proteasome complexes. Mol Cell Proteomics 2011; 10 : R110.006924. doi: 10.1074/mcp.M110.006924.

50 Scherz-Shouval R, Shvets E, Fass E, Shorer H, Gil L, Elazar Z. Reactive oxygen species are essential for autophagy and specifically regulate the activity of Atg4. EMBO J 2007; 26: 1749-60.

51 Pérez LM, Milkiewicz P, Ahmed-Choudhury J, Elias E, Ochoa JE, Sánchez Pozzi EJ, et al. Oxidative stress induces actin-cytoskeletal and tight-junctional alterations in hepatocytes by a $\mathrm{Ca}^{2+}$-dependent, PKC-mediated mechanism: protective effect of PKA. Free Radic Biol Med 2006; 40: 2005-17.

52 Son Y, Cheong YK, Kim NH, Chung HT, Kang DG, Pae HO. Mitogenactivated protein kinases and reactive oxygen species: How can ROS activate MAPK pathways? J Signal Transduct 2011; 2011: 792639. doi: 10.1155/2011/792639. 